\title{
BRAF-Mutated Erdheim-Chester Disease: Profound Response to Vemurafenib Visualized With Serial Multimodality Imaging
}

Javaughn Corey R. Gray, MS, MHS'; Jongho Kim, MD, PhD²; Michael Digianvittorio, DO2; Nancy K. Feeley, CRNP3; Paul J. Scheel, MD4; Stanley S. Siegelman, MD²; Elliot K. Fishman, MD²; and Steven P. Rowe, MD, PhD²

\begin{abstract}
Erdheim-Chester disease (ECD) is an extremely rare and aggressive non-Langerhans histiocytic disorder. ECD typically presents with bone pain in middle-aged adults, although some patients present with multisystem disease involving the skeleton, central nervous system, cardiovascular system, lungs, and other disease sites. The etiology of ECD is currently unknown, but it is thought to be a reactive or neoplastic disorder. Recently, mutation of the BRAF gene has been found in $>50 \%$ of ECD cases, and this gene has become a therapeutic target for patients with ECD. Vemurafenib, a BRAF inhibitor, has been approved by the FDA for treatment of ECD. This report presents an elderly male patient with an aggressive phenotype of ECD and highlights the utility of multimodality imaging in monitoring the clinical course and disease response to treatment with vemurafenib.
\end{abstract}

${ }^{1}$ Department of Oncology, Sidney Kimmel Comprehensive Cancer Center, ${ }^{2}$ The Russell H. Morgan Department of Radiology and Radiological Science, and ${ }^{3}$ Division of Nephrology, Department of Medicine, Johns Hopkins University School of Medicine, Baltimore, Maryland; and ${ }^{4}$ Division of Nephrology,

Washington University, Saint Louis, Missouri.
Erdheim-Chester disease (ECD) is a rare non-Langerhans histiocytic disorder typically characterized by multifocal osteosclerotic lesions of the long bones. ${ }^{1,2}$ Upon biopsy, these lesions commonly display sheets of foamy histiocytes. Histiocytic infiltration of extraskeletal tissues is also common. Indeed, ECD is a multisystem disease and almost all organs can be affected. Patients can present with skeletal, pulmonary, retroperitoneal, endocrine, neurologic, skin, renal, and cardiovascular involvement. ${ }^{3-5}$ The extent and distribution of disease determines the clinical course. Some patients present with only asymptomatic bone lesions, whereas others have multisystem, potentially life-threatening disease. As a result, the definitive diagnosis may be difficult to make because the clinical manifestations of ECD frequently overlap with those of other systemic fibroinflammatory disorders similar to ECD, such as Langerhans cell histiocytosis, retroperitoneal fibrosis, Paget disease, hemophagocytic lymphohistiocytosis, and Rosai-Dorfman disease.

ECD was first described by Erdheim and Chester in 1930, and since that time fewer than 500 cases have been reported in the literature. ${ }^{6}$ ECD predominantly affects adults with a median age of 55 years, and is more frequently diagnosed in men than women (3:1). ${ }^{7}$ The etiology of ECD remains unclear, but it is thought to be a hematopoietic neoplasm representing clonal proliferation of myeloid progenitor cells. This was discovered through investigation of $B R A F^{\mathrm{V} 600 \mathrm{E}}$ mutations in myeloid cells. ${ }^{8,9}$ Between $57 \%$ and $75 \%$ of patients with ECD carry the $B R A F^{\mathrm{V} 600 \mathrm{E}}$ mutation, an activating mutation of the proto-oncogene $B R A F^{10}$ This finding has had major implications on the treatment of ECD. Before this discovery, the main therapeutic approach to ECD was interferon- $\alpha$-based regimens. Now, there is increasing evidence that almost all patients carrying the $B R A F^{\mathrm{V} 600 \mathrm{E}}$ mutation have excellent responses to the now FDA-approved BRAF inhibitor vemurafenib and other BRAF/MEK-targeted inhibitors. ${ }^{2,11}$ In a recent study of patients with ECD neurologic involvement, a combination of anatomic MRI and metabolic imaging 
with FDG-PET showed a high rate of objective response to targeted therapies such as vemurafenib. ${ }^{12}$

This report presents a case of ECD in a 70-year-old man, with a focus on the extensive imaging findings at baseline and of the clinical course after initiation of treatment with vemurafenib.

\section{Case Summary}

A 70-year-old white man with a past medical history significant for hypertension, chronic kidney disease, anemia, and pericardial effusion initially presented with severe fatigue, cognitive impairment, and diffuse bone pain. He also reported muscle pain in his thighs and calves, diminished appetite, and severe lethargy. In a prior visit for pericardial effusion treatment, an abdominal CT study reportedly showed mild enlargement of the kidneys with marked diffuse cortical thickening bilaterally and symmetrically, and multiple ill-defined soft tissue nodules in the omentum and abdomen. Biopsy of the perirenal fascia revealed a fibroinflammatory process with no evidence of lymphoma and negative stains for immunoglobulin G4. The patient opted for outside workup and ultimately returned for a neurology consult. A brain MRI scan reportedly showed bilateral inflammatory retro-orbital masses. Due to failure to find a diagnostic solution for his problems, he was admitted to our institution and a more extensive workup was performed.

Initial laboratory evaluation revealed microcytic hypochromic anemia with a hemoglobin level of $9.1 \mathrm{~g} / \mathrm{dL}$. All other baseline laboratory values were within normal limits. Multisystem symptoms prompted a workup including imaging, with whole-body technetium-99mmethylene diphosphonate $\left.{ }^{99 \mathrm{~m}} \mathrm{Tc}-\mathrm{MDP}\right)$ bone scan showing markedly increased radiotracer uptake in the metadiaphyses of the right and left distal femora and proximal tibiae (Figure 1). More moderate increases of uptake were seen bilaterally in the distal metadiaphyses of the tibiae and radii. These findings, including the distribution of uptake, suggested the possibility of ECD.

Follow-up bilateral radiograph of the knees showed a diffuse sclerotic appearance of bilateral distal femoral and proximal tibial metaphysis with foci of lucency, endosteal thickening, and bilateral solid periosteal reaction (Figure 2). This constellation of radiographic findings was, again, suggestive of ECD with symmetric involvement of lower extremities.

FDG-PET/CT was performed to evaluate the metabolic profile of the probable ECD (Figures 3-5). Extensive renal and perirenal involvement was visualized, with extensive infiltrative soft tissue surrounding the kidneys and bilateral stranding with extensive soft tissue induration at the renal hilum (see Figure 3). Similarly,
A

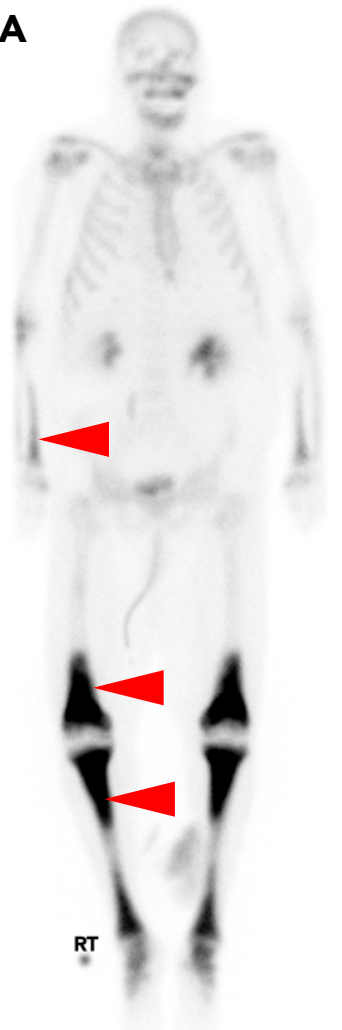

B
Figure 1. Whole-body $(A)$ anterior and $(B)$ posterior images from the patient's baseline $99 \mathrm{~m} T \mathrm{Tc}-\mathrm{MDP}$ bone scan show intense, metadiaphyseal, abnormal radiotracer uptake in multiple long bones of the appendicular skeleton (red arrowheads). This highly unusual pattern of uptake is typical for Erdheim-Chester disease.

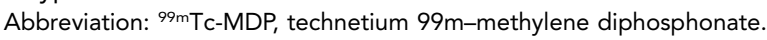

moderate hydronephrosis and a marked increase of metabolic activity was noted on PET. Orbital, extraconal findings, also consistent with ECD, were apparent on PET/ CT; infiltrative soft-tissue thickening within the orbits was present, with associated intense FDG uptake (see Figure 4).

The FDG-PET/CT scan also revealed cardiac findings, which were further investigated with cardiac MRI (see Figure 5). Infiltrative, nodular, and enhancing mass-like involvement of the right atrial wall and right atrioventricular groove was present. This finding, when combined with additional sites of involvement, including the bilateral orbits, perinephric spaces, and skeletal structures, created a picture consistent with ECD. A bone biopsy of the distal left femur, performed concurrent with the imaging studies, was positive for extensive marrow fibrosis and histiocyte inflammation, confirming the diagnosis.

Due to the presence of histiocytes in biopsy specimens, $B R A F$ mutation analysis was ordered, which was positive for the $B R A F^{\mathrm{V} 600 \mathrm{E}}$ mutation. Because of the disease severity, initial treatment with anakinra (100 mg/d subcutaneous) for bone pain and other 

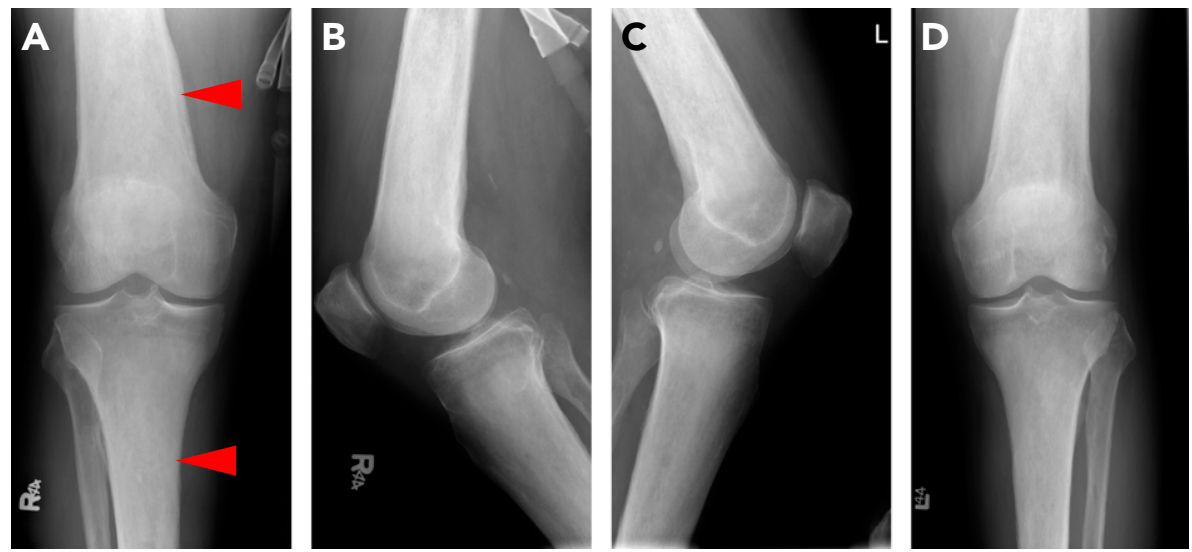

Figure 2. Follow-up radiographs of the right knee ([A] anteroposterior and $[B]$ lateral) and left knee ([C] lateral and [D] anteroposterior) show dense sclerosis in the visualized femoral and tibial metadiaphyses (red arrowheads), corresponding to the abnormal uptake on bone scan and typical for Erdheim-Chester disease.

constitutional symptoms was initiated while awaiting biopsy results, and the patient experienced immediate improvement in fatigue and bone pain and improved cognition, energy, ambulation, and pain control. Furthermore, the patient's anemia resolved and erythrocyte sedimentation rate decreased after treatment initiation. Following this initial response, and upon confirmation of the $B R A F$ mutation, concurrent treatment with vemurafenib (240 mg twice per day) was initiated. Vemurafenib treatment was associated with manageable adverse effects, including verrucous keratoses, pruritus, and leukonychia.

As shown in Figures 3-5, treatment with vemurafenib led to anatomic and metabolic resolution of all abnormal imaging findings. Scans progressively showed increased resolution of right atrial FDG uptake and resolution of the perinephric soft tissue rind and hydronephrosis. By 4 years after initial presentation, FDG-PET/CT scans noted complete metabolic response
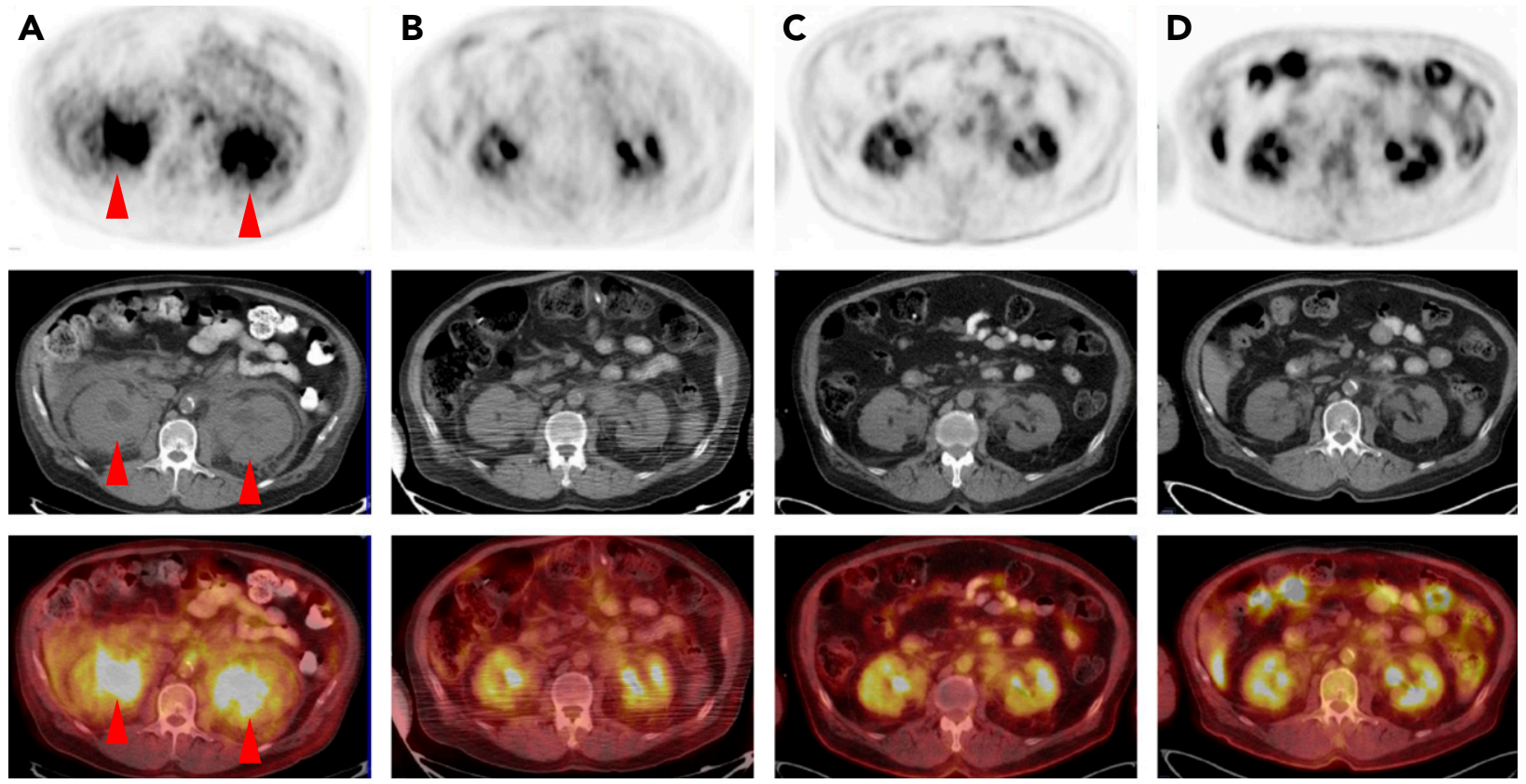

Figure 3. Axial PET (top row), CT (middle row), and PET/CT (bottom row) images through the upper abdomen at (A) baseline and (B) 6 months, (C) 2 years, and (D) 4 years after initiation of therapy. The baseline scan is notable for metabolically active, extensive soft tissue surrounding the kidneys, with marked thickening of the perirenal fascia, enlargement of the kidneys, and hydronephrosis (red arrowheads). These findings (B) had nearly resolved 6 months after the patient started therapy and $(C$ and $D)$ continued to normalize on serial imaging over the next 4 years. 

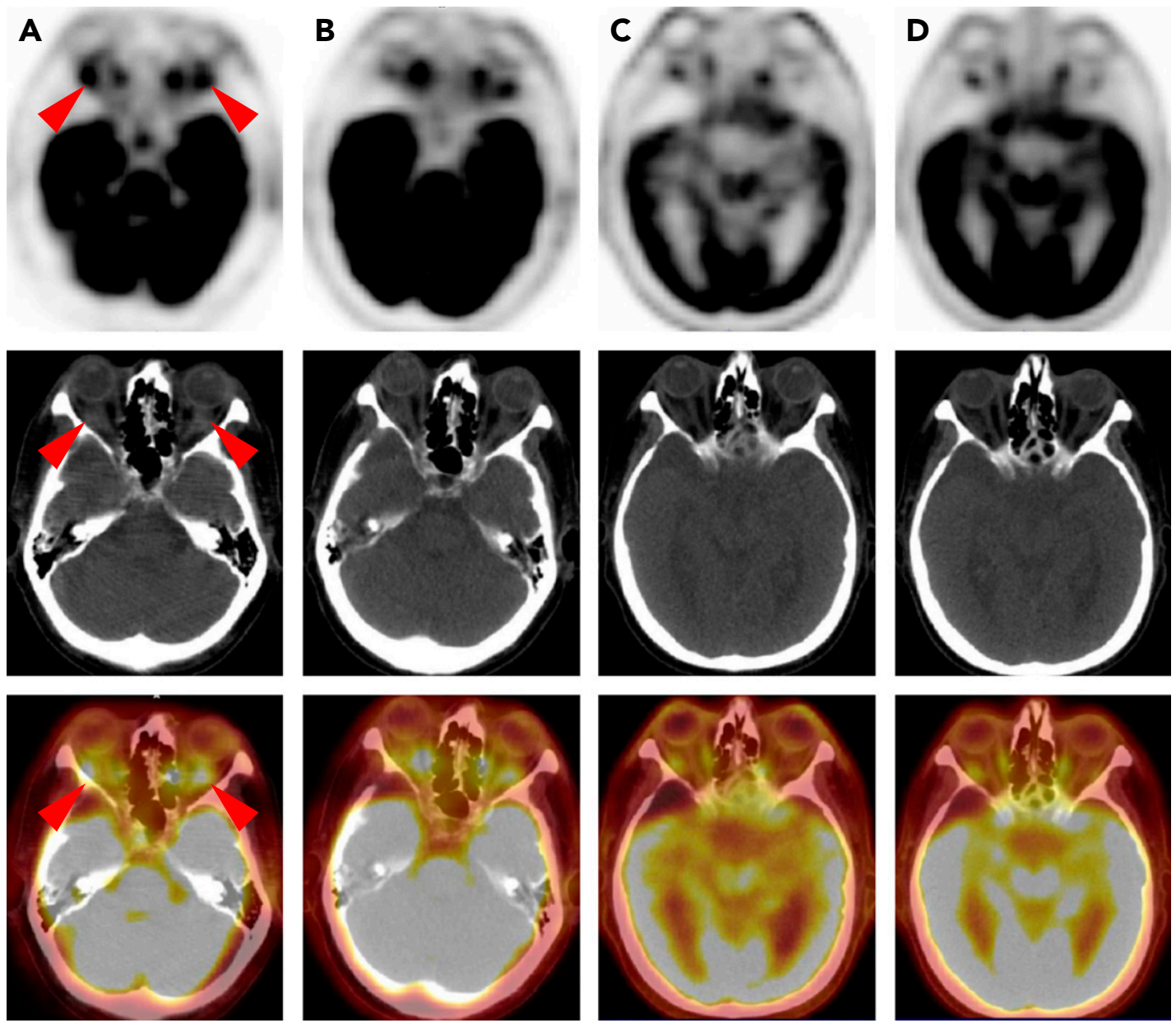

Figure 4. Axial PET (top row), CT (middle row), and PET/CT (bottom row) images through the brain and orbits at (A) baseline and (B) 6 months, (C) 2 years, and (D) 4 years after initiation of therapy. Note the intensely hypermetabolic soft tissue in the orbits at baseline (red arrowheads), with (B) persistence of the findings at the 6-month time point, and (C and D) then resolution of metabolic activity with only subtle residual orbital soft tissue at later times.

and decrease in extent of disease as noted by nonFDG-avid perinephric soft tissue stranding, mottled lesions in the distal femora and proximal tibiae, and homogeneous diffuse FDG uptake in the left ventricle myocardium (physiologic), with no FDG activity in the right ventricular myocardium. No new or metabolically active lesions or recurrence were noted elsewhere in the body.

\section{Discussion}

ECD is typically difficult to diagnose on imaging because of its effects on multiple systems and variable manifestations, its potentially nonspecific findings that can be associated with other disorders, and its rarity. To date, there has been no consensus on a diagnostic paradigm for ECD.

In this patient, a left tibial biopsy revealed the $B R A F^{\mathrm{V} 600 \mathrm{E}}$ mutation. Mutational analysis combined with findings of imaging studies, including histiocytosis in the retro-orbital space, right atria, pericardium, and perinephric space, supported the final diagnosis of ECD. Presence of the targetable BRAF mutation in the patient's histiocytes allowed for precise treatment to be delivered. Because mutation of the BRAF gene allows for unregulated production of tyrosine kinase and ultimately uncontrolled cellular proliferation, the $B R A F$-inhibitor vemurafenib was selected for this patient. At the time of diagnosis and treatment in this patient, there were no FDA-approved treatments for ECD, although clinical experience supported the use of vemurafenib in patients with BRAF-mutated ECD. ${ }^{13}$ The profound response to therapy visualized on imaging in this patient concords with the eventual FDA approval of vemurafenib in this context.

Anatomic and metabolic involvement in 4 characteristic organ systems (cardiac, renal, bone, and orbital) was initially noted, and subsequently followed, using serial multimodal imaging. A combination of radiograph, bone 
A
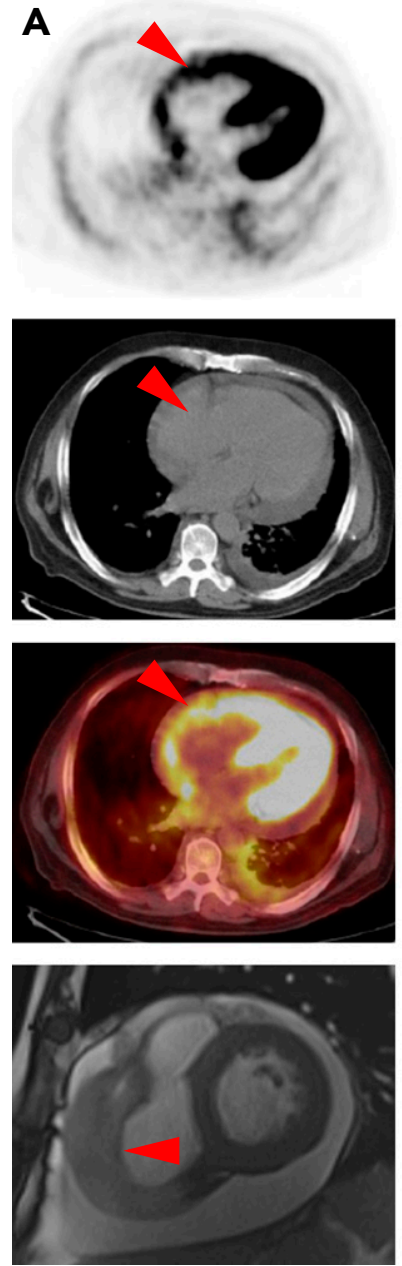

B
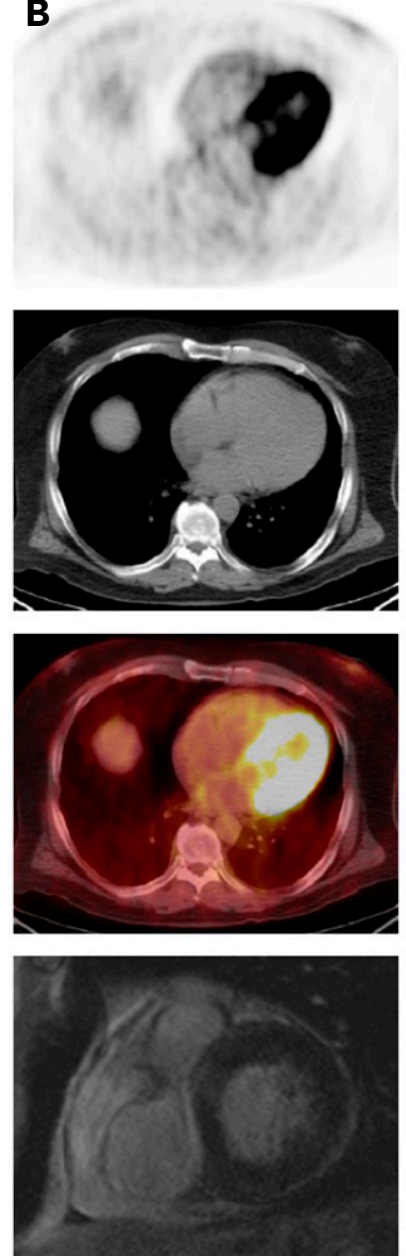
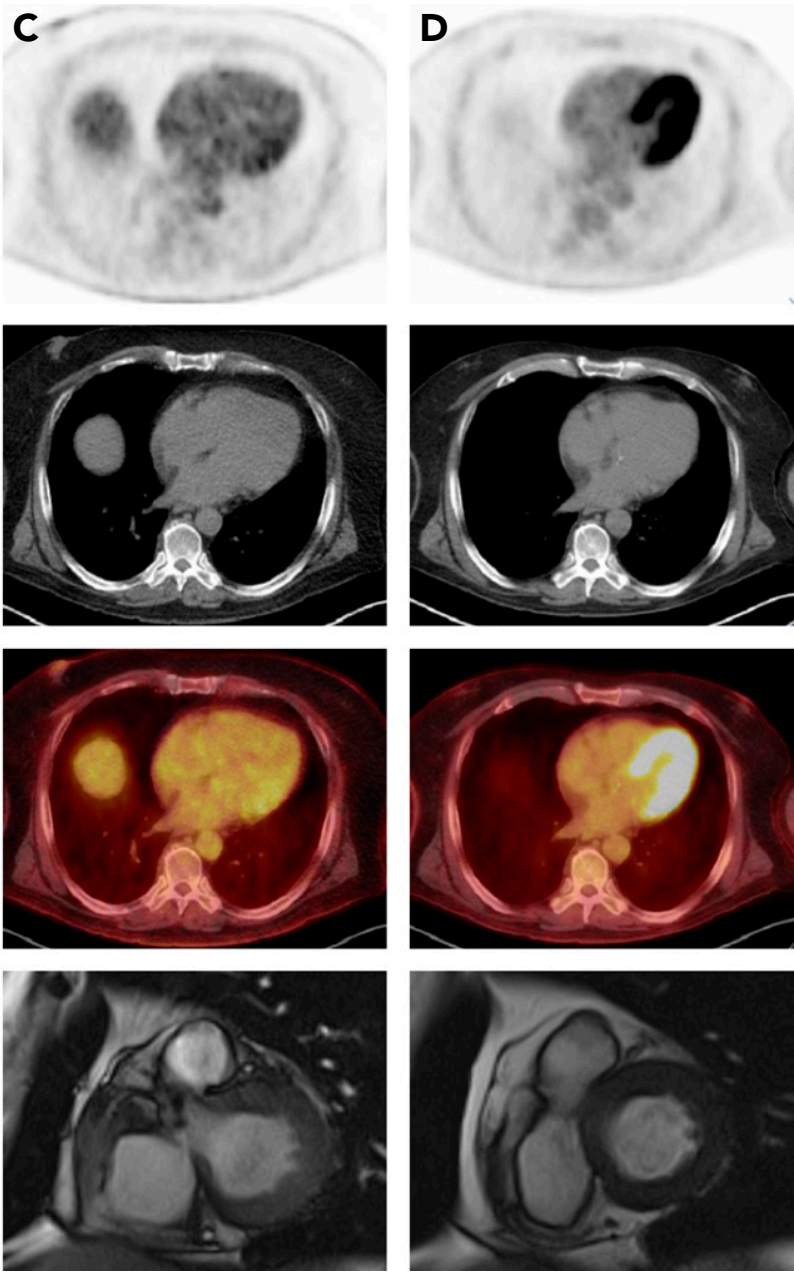

Figure 5. Axial PET (top row), CT (second from top row), and PET/CT (second from bottom row) images through the chest and short-axis, brightblood cardiac MRI (bottom row) images at (A) baseline and (B) 6 months, (C) 2 years, and (D) 4 years after initiation of therapy. FDG uptake on PET in the left ventricle is variable, but often physiologic. However, intense uptake in the right ventricle and right atrium, as seen here in the patient's baseline images, is atypical (red arrowheads). This abnormal metabolic activity corresponds to marked thickening on the cardiac MRI (red arrowhead). Metabolic activity in this region had nearly normalized by the 6-month follow-up images, although the thickening of the right ventricular wall was similar on MRI. By 2 and 4 years after initiation of therapy, the FDG uptake on PET had completely resolved with a gradual decrease in the abnormal right ventricular thickening on MRI.

scan, MRI, and PET/CT provided evidence of metabolic activity of histiocytes and locations of active lesions. Radiographically, we noted a complete response to vemurafenib treatment with complete normalization of anatomic and metabolic involvement. This response was durable, with no evidence of recurrent, metabolically active disease 4 years after the patient's initial presentation.

\section{Conclusions}

This case suggests that mutational analysis should be performed if imaging findings are highly suggestive of ECD and response can be monitored through serial imaging. It is likely that in most patients with multisystem disease, a combination of imaging modalities is necessary to best assess disease status. For example,
FDG-PET/CT has the advantage of providing metabolic information and can provide important characterization information for persistent anatomic abnormalities. However, in regions of the body that have intrinsic high or variable physiologic uptake of FDG (such as structures near the brain or myocardium), anatomic changes on MRI may be more reliable.

Submitted October 10, 2019; accepted for publication February 18, 2020.

Disclosures: The authors have disclosed that they have not received any financial consideration from any person or organization to support the preparation, analysis, results, or discussion of this article

Correspondence: Steven P. Rowe, MD, PhD, Division of Nuclear Medicine and Molecular Imaging, The Russell H. Morgan Department of Radiology and Radiological Science, Johns Hopkins University School of Medicine, 600 North Wolfe Street, Baltimore, MD 21287. Email: srowe8@jhmi.edu 


\section{References}

1. Campochiaro C, Tomelleri A, Cavalli G, et al. Erdheim-Chester disease. Eur J Intern Med 2015;26:223-229.

2. Haroun F, Millado K, Tabbara I. Erdheim-Chester disease: comprehensive review of molecular profiling and therapeutic advances. Anticancer Res 2017;37:2777-2783.

3. Diamond EL, Dagna L, Hyman DM, et al. Consensus guidelines for the diagnosis and clinical management of Erdheim-Chester disease. Blood 2014; 124:483-492.

4. Arnaud L, Hervier B, Néel A, et al. CNS involvement and treatment with interferon- $\alpha$ are independent prognostic factors in Erdheim-Chester disease: a multicenter survival analysis of 53 patients. Blood 2011;117:2778-2782.

5. Haroche J, Amoura Z, Dion E, et al. Cardiovascular involvement, an overlooked feature of Erdheim-Chester disease: report of 6 new cases and a literature review. Medicine (Baltimore) 2004;83:371-392.

6. Cavalli G, Guglielmi B, Berti A, et al. The multifaceted clinical presentations and manifestations of Erdheim-Chester disease: comprehensive review of the literature and of 10 new cases. Ann Rheum Dis 2013;72:1691-1695.

7. Emile JF, Abla O, Fraitag S, et al. Revised classification of histiocytoses and neoplasms of the macrophage-dendritic cell lineages. Blood 2016;127: 2672-2681
8. Milne $\mathrm{P}$, Bigley $\mathrm{V}$, Bacon $\mathrm{CM}$, et al. Hematopoietic origin of Langerhans cell histiocytosis and Erdheim-Chester disease in adults. Blood 2017;130: 167-175.

9. Durham BH, Roos-Weil D, Baillou C, et al. Functional evidence for derivation of systemic histiocytic neoplasms from hematopoietic stem/ progenitor cells. Blood 2017;130:176-180.

10. Haroche J, Papo M, Cohen-Aubart F, et al. Erdheim-Chester disease (ECD), an inflammatory myeloid neoplasia [in French]. Presse Med 2017 46:96-106.

11. Diamond EL, Subbiah V, Lockhart AC, et al. Vemurafenib for BRAF V600mutant Erdheim-Chester disease and Langerhans cell histiocytosis: analysis of data from the histology-independent, phase 2, open-labe VE-BASKET Study. JAMA Oncol 2018;4:384-388.

12. Bhatia A, Hatzoglou V, Ulaner G, et al. Neurologic and oncologic features of Erdheim-Chester disease: a 30-patient series [published online January 17, 2020]. Neuro-oncol, doi: 10.1093/neuonc/noaa008

13. Haroche J, Cohen-Aubart F, Emile JF, et al. Dramatic efficacy of vemurafenib in both multisystemic and refractory Erdheim-Chester disease and Langerhans cell histiocytosis harboring the BRAF V600E mutation. Blood 2013;121:1495-1500.

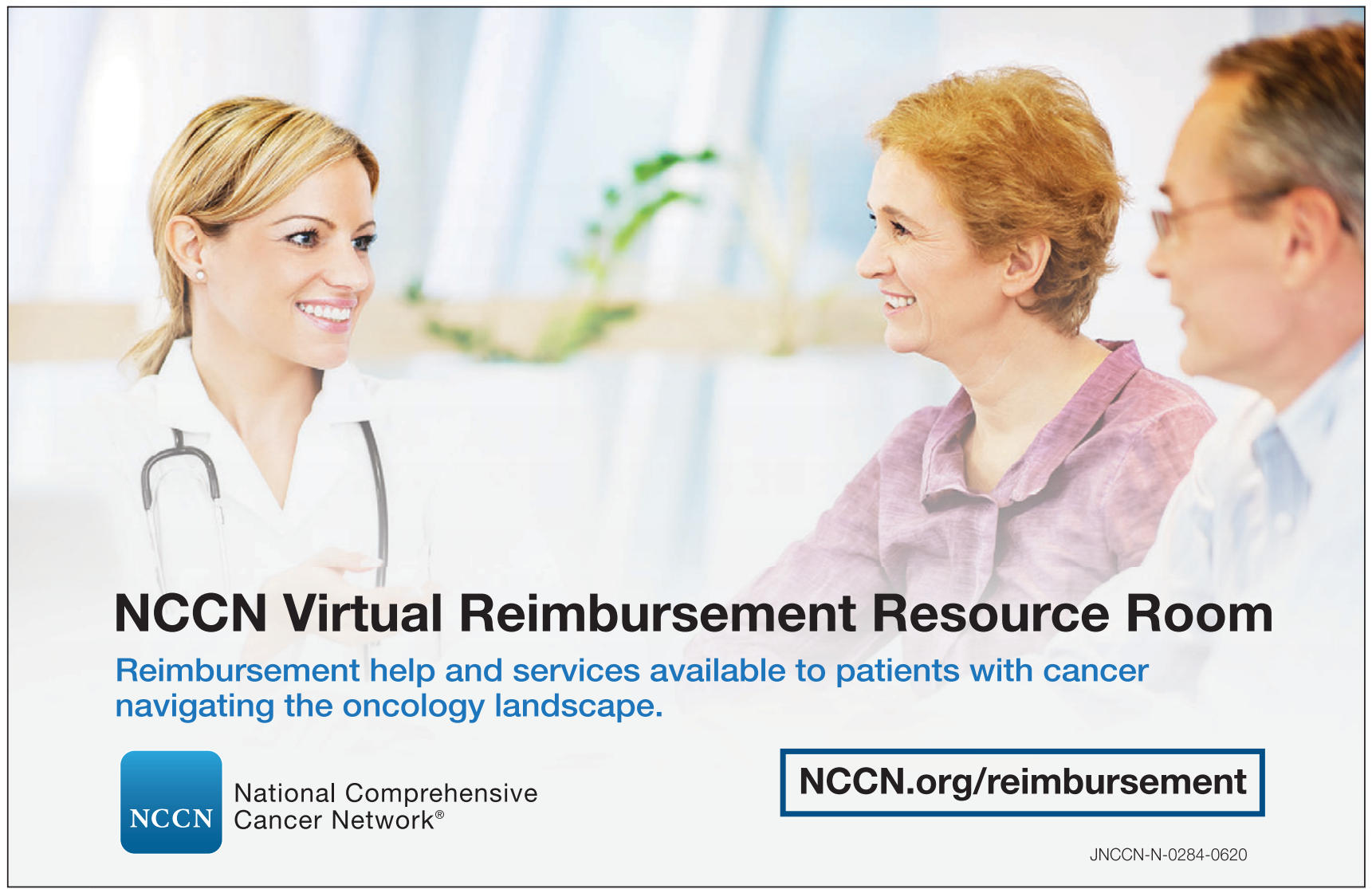

